\title{
The sub-seasonal to seasonal prediction project (S2S) and the prediction of extreme events
}

\author{
Frédéric Vitart ${ }^{1}$ and Andrew W. Robertson ${ }^{2}$
}

The sub-seasonal to seasonal prediction project (S2S) is a 5-year project, established in 2013 by the World Weather Research Program (WWRP) and the World Climate Research Program (WCRP). This paper briefly describes the S2S project in the context of extended range prediction of extreme events. We provide evidence that S2S forecasts have the potential to predict the onset, evolution and decay of some large-scale extreme events several weeks ahead. For instance, S2S models displayed skill to predict high probabilities of extreme 2-m temperature anomalies over Russia during the worst week of the 2010 Russian heat wave up to 3 weeks in advance. In other cases, like for tropical cyclone prediction, S2S models can produce useful information on the probability of the occurrence of tropical storms within sufficiently large areas through the prediction of large-scale predictors, such as the Madden-Julian Oscillation (MJO). In future, S2S forecasts of extreme events could be integrated into a "ready-set-go" framework between seasonal and medium range forecasts, by providing an early warning of an extreme event a few weeks in advance. Finally, S2S forecasts can also be used to investigate the causality of some extreme events and we show evidence that the cold March 2013 over western Europe and North Asia was linked to a MJO event propagating over the western Pacific.

npj Climate and Atmospheric Science (2018)1:3 ; doi:10.1038/s41612-018-0013-0

\section{INTRODUCTION}

There is increasing interest in extreme weather and climate events, both in order to develop early warning systems to improve societal preparedness, as well as to gain a better understanding of the impacts of climate change. Extreme weather and climate events pose a serious threat to the health and welfare. For instance, between 2011 and 2013, the United States experienced 32 weather events that each caused at least one billion dollars in damage, with a total of more than $\$ 110$ billion in damages in 2012 $\left(N O A A^{1}\right)$. According to Munich $\mathrm{Re}^{2}$ the world's largest reinsurance company, more than 90 percent of all disasters and 65 percent of associated economic damages in 2010 were weather and climate related (i.e., high winds, flooding, heavy snowfall, heat waves, droughts, and wildfires), although there were far more deaths from geological disasters that year (almost entirely from the Haiti earthquake). In all, 874 weather and climate-related disasters resulted in 68,000 deaths and $\$ 99$ billion in damages worldwide in 2010. These extreme weather events can also damage critical infrastructure, such as roads, railways or power and telecommunication grids. For instance, during the latest flooding in the United Kingdom at the end of 2015, some 20,000 homes were left without power. Therefore, the prediction of weather extremes is one of the major challenges of weather forecasting and one of the main duties of national services to allow appropriate mitigating action to be taken and contingency plans to be put into place by the authorities and the public.

Sub-seasonal to seasonal forecasting (defined here as the time range between 2 weeks and 2 months) bridges the gap between the more-mature weather and seasonal climate prediction communities. It has received much less attention than medium range and seasonal prediction despite the considerable socio- economic value that could be derived from such forecasts. This time range is critical for pro-active disaster mitigation efforts, since they may take several weeks to implement. ${ }^{3}$ It is considered a difficult time range since the lead time is sufficiently long that much of the memory of the atmospheric initial conditions is lost and it is too short for the variability of the ocean to have a strong influence. However, recent research has indicated important potential sources of predictability for this time range such as the MJO, the evolution of ENSO, soil moisture, snow cover and sea ice, stratosphere-troposphere interactions, ocean conditions and tropical-extratropical teleconnections. ${ }^{4}$

The sub-seasonal to seasonal prediction project (S2S) project and database will be briefly described in The S2S project and database section. Types of extreme weather and climate prediction section will discuss the type of extreme event prediction we could expect for this time range. Sub-seasonal prediction of tropical cyclones section will present examples of tropical cyclone sub-seasonal predictions and Prediction of the 2010 Russian heat wave section will discuss the sub-seasonal prediction of the Russian heat wave of 2010. Finally, Attribution of extreme events section will discuss the possible use of sub-seasonal forecasts for the attribution of some extreme event.

\section{THE S2S PROJECT AND DATABASE}

To bridge the gap between medium range weather forecasts and seasonal forecasts, in 2013 the World Weather Research program (WWRP) and the World Climate Research program (WCRP) jointly launched a 5-year research initiative called the S2S project. Its goal is to improve forecast skill and understanding of the sources of subseasonal to seasonal predictability, and to promote its uptake by

\footnotetext{
${ }^{1}$ European Centre for Medium-Range Weather Forecasts (ECMWF), Reading, UK and ${ }^{2}$ International Research Institute for Climate and Society (IRI), Columbia University, Palisades, NY, USA

Correspondence: Frédéric Vitart (nec@ecmwf.int)
}

Received: 19 May 2017 Revised: 15 September 2017 Accepted: 15 September 2017

Published online: 12 March 2018 
operational centers and exploitation by the applications communities (www.s2sprediction.net). The research is organized around a set of six topics (Madden-Julian Oscillation (MJO), Monsoons, Africa, Extremes, Teleconnections and Verification), each being intersected by the cross-cutting research and modeling issues, and applications and user needs. ${ }^{5}$ To address these issues, the S2S project has created an extensive database ${ }^{6}$ (the S2S Database) containing subseasonal (up to 60 days) forecasts and reforecasts (sometimes known as hindcasts) from 11 operational and research centers. It is modeled in part on the THORPEX Interactive Grand Global Ensemble (TIGGE) database for medium range forecasts (up to 15 days) $^{7}$ and the Climate-System Historical Forecast project (CHFP) (http://wcrp-climate.org/index.php/wgsip-chfp/chfp-overview) for seasonal forecasts. This database is archived at the European Centre for Medium range Weather Forecasts (ECMWF), and the Chinese Meteorological Administration (CMA) and (in part) at the International Research Institute for Climate and Society (IRI) and provides an important community resource for research on the predictability of extreme weather.

\section{TYPES OF EXTREME WEATHER AND CLIMATE PREDICTION}

Historically, numerical prediction of extreme events started in the 1960 s with the emergence of computers and operational numerical weather forecasting. It was originally exclusively a short-range deterministic forecasting problem, with the goal of predicting specific extreme weather events a few minutes to a few days in advance. On the other hand, with the advent of dynamical seasonal forecasts in the 1990s, probabilistic forecasts of extreme events such as tropical storm frequency at the ocean-basin scale were developed in the early 2000s, 8 and extreme weather became an increasingly important research topic for climate change projection. In the case of climate prediction, the main goal is to determine how much seasonal-to-interannual climate variability or climate change will affect the statistics (frequency, intensity, duration..) of extreme weather events (see for example Knutson et al. ${ }^{10}$ for the impact of global warming on tropical cyclone activity). Therefore, short/medium range and climate forecasting of extreme weather events answer very different questions, the former being a (largely atmospheric) initial value problem for specific weather phenomena such as tropical or extratropical cyclones, while the latter is largely an atmospheric boundary condition problem for the spatio-temporal statistics of individual extreme events, depending, for example, on sea surface temperatures, or on greenhouse gas concentrations, respectively. The latter are inherently probabilistic, while a probabilistic treatment of weather forecasts is more recent. Sub-seasonal to seasonal prediction, which lies in between, is a combination of both, being an initial value as well as a boundary condition problem. Depending on the nature and predictability of a specific extreme weather event, sub-seasonal to seasonal forecasting systems could be used to predict a specific extreme weather event at longer lead time than a weather forecast, or to predict changes to the statistics of the event in the coming few weeks or months compared to climatology. Small scale, short lived extreme weather events such as tornadoes which have a predictability of the order of minutes cannot be predicted individually weeks in advance, but it may be possible to predict changes to probability of tornado activity over large areas and windows of time due to their relationship to large-scale weather circulation. It may also be possible to predict the genesis, duration and decay a few weeks in advance of a large scale, long-lasting extreme weather events, such as heat waves or droughts. Therefore, depending on the nature of the extreme weather/climate event, S2S prediction may look more like an initial value problem such as in short/medium range forecasting of specific large-scale long-lived events, or more like a boundary condition problem for event statistics such as in seasonal forecasting or climate change projection.

\section{SUB-SEASONAL PREDICTION OF TROPICAL CYCLONES}

Short and medium range forecasts of tropical storm track and intensity have been available for a few decades. These forecasts predict the trajectory of tropical cyclones which are already present in the initial conditions (e.g., Kurihara et al., ${ }^{11}$ ) and more recently also from tropical storms which are predicted to develop in a few days. ${ }^{12}$ More recently, seasonal forecasts of tropical storms have been developed (e.g., Vitart and Stockdale ${ }^{8}$ ) which predict if a tropical cyclone season over a specific basin will be more or less active than normal. These seasonal forecasts are justified by the fact that tropical cyclone genesis is sensitive to its large-scale environment, most especially the vertical wind shear between the upper and lower troposphere, mid-level humidity, low level vorticity. ${ }^{13}$ As a consequence, the El-Niño Southern Oscillation (ENSO) and local sea surface temperatures (SSTs) which affect these environmental parameters play a major role in modulating tropical cyclone activity over some ocean basins.

S2S which lies in between medium range and seasonal time ranges can include both aspects of tropical cyclone prediction. There are cases where extended range prediction of a specific tropical storm might be possible if the genesis can be predicted a few days in advance (e.g., tropical cyclone Nargis over the North Indian Ocean in $2010^{14}$ ) combined to the fact that some tropical storms can last for more than 3 weeks. However, successful tropical storm track forecast beyond 2 weeks remains very rare, despite significant improvement in short and medium range tropical storm track forecasting over the past decades. At the S2S time scale, most of the tropical storm predictability is likely to be provided by changes to the large-scale circulation which affect tropical cyclone activity as for seasonal forecasting. ENSO and Indian Ocean Dipole (IOD) can be a source of predictability for tropical cyclones in some ocean basins as for seasonal forecasting and are indeed used as predictors for statistical sub-seasonal forecast models. ${ }^{15}$ However, the main source of predictability for tropical storm activity at the sub-seasonal time scale is often the MJO (e.g., Nakazawa, ${ }^{16}$ ) particularly over the Southern Hemisphere where the tropical cyclone season coincide with the strongest MJO activity (boreal winter and spring) through mostly its impact on low level absolute vorticity and vertical wind shear. ${ }^{17}$ The modulation of tropical cyclone numbers by the phase of the MJO has been quoted to be as high as 4:1 in some locations (e.g., Maloney and Hartmann. ${ }^{18}$ ) Other sources of predictability at the intra-seasonal time scale include equatorial Rossby (ER) waves, mixed Rossby gravity (MRG) waves, easterly waves, extratropical waves, and equatorial Kelvin wave. ${ }^{19}$

In order to be skillful in the sub-seasonal prediction of tropical cyclones, it is therefore essential for S2S models to display skill in predicting these various sources of sub-seasonal predictability, and most especially the MJO. The skill of sub-seasonal forecasts to predict the MJO has been assessed in the S2S database using the Wheeler and Hendon MJO index. ${ }^{20,21}$ This index consists of projecting the model forecasts onto the first two EOFs of $850 \mathrm{hPa}$, $200 \mathrm{hPa}$ zonal wind and outgoing long-wave radiation (OLR), precomputed from NCEP/NCAR reanalysis for winds and satellite observations for OLR. A bivariate correlation ${ }^{22}$ of the two principal component time series from the model forecasts and ERA Interim is then computed. Figure 1 shows the skill of the models from the S2S databases to predict the MJO for the common re-forecast period 1999-2010, measured here as the forecast day when the bivariate correlation reaches 0.5 or 0.6 . This figure shows that the S2S models display some skill to predict the MJO up to about 3 weeks on average, and for the ECMWF model beyond 4 weeks. Model experimentation would be needed to determine which specific aspect of the forecasting system (initialization, ensemble generation, model physics, model resolution..) could explain this lead. However, specific changes to the ECMWF model physics, ${ }^{23}$ 


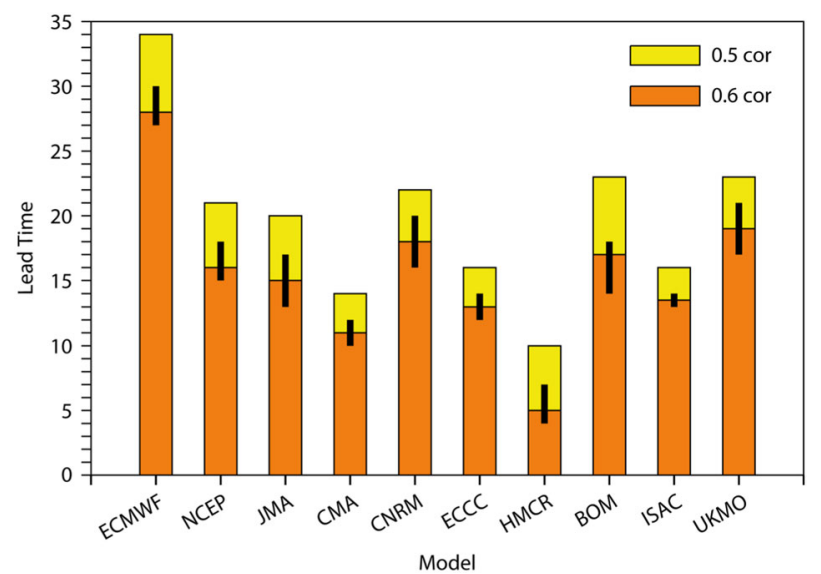

Fig. 1 MJO forecast skill. Forecast lead time (days) when the MJO bivariate correlation reaches 0.5 (yellow bars) or 0.6 (orange bars) for 10 model re-forecasts from the S2S database covering the common period 1999-2010. The black vertical bars represent the $10 \%$ level of confidence for a bivariate correlation of 0.6 using a 10,000 resampling bootstrap technique

especially in the convective parameterization helped to increase the limit of MJO predictive skill by 2 weeks over the past decade.

Demonstrating skill in predicting the MJO is an important step for sub-seasonal prediction of tropical cyclones, but it is also important for the dynamical models to be able to simulate the impact of the MJO on the tropical cyclones that are produced by the model. In order to assess if S2S models can reproduce this modulation of tropical cyclone activity by the MJO, the modelsimulated tropical cyclones have been tracked ${ }^{24}$ in each model ensemble forecast. Since the S2S database output are gridded at a 1.5-degree resolution, the model tropical cyclones tend to be weaker than observed. The threshold for 10-meter maximum wind has been adjusted for each model so that the total climatological density of simulated tropical cyclones matches the observations. Longer model integrations would be needed to assess the realism of other characteristics of simulated tropical cyclones (e.g. tropical cyclone interannual variability). This tracking has been applied to eight S2S model reforecasts which were chosen because of their large re-forecast frequency and ensemble size and their skill to predict the evolution of the MJO: ECMWF, NCEP, JMA, BOM, UKMO, CMA, ECCC and CNRM. Work is ongoing to extend this study to the other S2S models. The density of tropical cyclone tracks (number of tropical cyclones passing within $500 \mathrm{~km}$, normalized by the total number of tropical storms over the whole basin) has been calculated for each model. All eight S2S models display more (less) tropical cyclone activity over the Indian Ocean and less (more) tropical cyclone activity over the South Pacific and near the Maritime Continent when there is an MJO in phase 2 or 3 (6 or 7) in the model (Fig. 2), which is consistent with observational studies $^{17}$ and with previous modeling studies. ${ }^{25}$ This result suggests that the models are capable of reproducing very well the modulation of tropical cyclones in the southern Hemisphere by the MJO, even if the model resolution is very coarse (BoM has a resolution of the order of $200 \mathrm{~km}$ ). Therefore, even if the dynamical models considered in Fig. 2 are not able to predict the occurrence of a given storm at a precise location 3 to 4 weeks in advance, they are likely to have some skill in predicting an increase or decrease of tropical cyclone activity over a large domain and a sufficiently large period of time. For example at ECMWF sub-seasonal forecasts of tropical cyclone activity are produced over weekly mean periods and each ocean basin. Verification of these forecasts ${ }^{26,27}$ suggests some skill up to at least 2 weeks over most of the basins, and up to week 3 over the South Indian ocean.
Extending upward from the more-mature medium range weather forecasting of individual tropical storms, the results above suggest that there is a potential opportunity to extend tropical cyclone forecasting to longer lead times, using probabilistic forecasts of tropical cyclone density or landfall. In the context of humanitarian aid and disaster preparedness, the Red Cross Climate Centre/IRI have proposed a "Ready-Set-Go" early-warning concept for taking action based on forecasts from weather to seasonal, in which seasonal forecasts are used to begin monitoring of sub-seasonal and short-range forecasts, update contingency plans, train volunteers, and enable early warning systems ("Ready"); sub-monthly forecasts would be used to alert volunteers, warn communities ("Set'); and, weather forecasts are then used to activate volunteers, distribute instructions to communities, and evacuate if needed ("Go"). This seamless forecasts to action paradigm could be applied to tropical cyclones prediction. Figure 3 shows an example of Ready-Set-Go paradigm for the prediction of tropical cyclone Yasi, which made landfall in northern Queensland, Australia on 3 February 2011, as a severe Category 5 causing major damage to affected areas. The storm caused an estimated AU\$3.5 billion (US $\$ 3.6$ billion) in damage, making it the costliest tropical cyclone to hit Australia on record (source https://en.wikipedia.org/wiki/Cyclone_Yasi). Figure 3 suggests that sub-seasonal forecasts could have provided useful information in a seamless prediction of tropical cyclone activity from seasonal forecasts. At the seasonal time scale (ready), the model predicted, as early as $1^{\text {st }}$ November, that the DecemberMarch tropical cyclone season in the Australian basin would likely be more active than normal, and that this signal was statistically significant within the $10 \%$ level of confidence. This seasonal forecast was consistent with La Niňa conditions, which prevailed during the 2010-2011 austral summer (more tropical cyclone activity over the Australian basin and less tropical cyclones in the South Pacific). At the sub-seasonal time scale, the forecast issued on 13 January for the 26 January-4 February period predicted $20-30 \%$ chance of tropical cyclone landfall in the Queensland area, which is well above the climatological probability, adding more geographical and temporal specificity to the forecast of a landfall, and increasing its confidence. At the medium range time scale, the probability of landfall from a 5-12 day forecast issued on January 27 reaches $90 \%$ which should trigger some action such as activating volunteers, distributing instructions to communities, and evacuating if needed. This type of seamless forecasts could be a possible contribution of sub-seasonal forecasts to climate service development within the Global Framework for Climate Services (GFCS).

\section{PREDICTION OF THE 2010 RUSSIAN HEAT WAVE}

Long-lasting heat waves, which can last from a week to several months enter into the category of extreme climate events where sub-seasonal forecast could potentially be used to predict the onset, evolution and decay a few weeks in advance. This section will discuss the predictability of a specific heat wave event: the 2010 Russian heat wave. This heat wave was the strongest ever recorded over the past 30 years. ${ }^{28}$ It caused an estimated 55,000 deaths and caused wildfires, the worst drought over Russia in nearly 40 years and the loss of at least millions hectares of crops.

The heat wave which lasted a few months (May-August 2010), was particularly intense during the week of 1-7 August 2010, where the weekly 2-m temperature anomalies over Russia reached a record value of $+5 \mathrm{C}$ (exceeding the heat wave over France in 2003). Re-forecasts from the S2S database have been used to assess the capability of state-of-the-art extended range forecasts to predict this specific event. 2-m temperature anomalies have been computed relative to the model climatology from 1999 to 2009 and averaged over the area $20 \mathrm{E}-50 \mathrm{E}, 45 \mathrm{~N}-70 \mathrm{~N}$ where this event took place. According to Fig. 4, ECMWF ensemble forecasts 

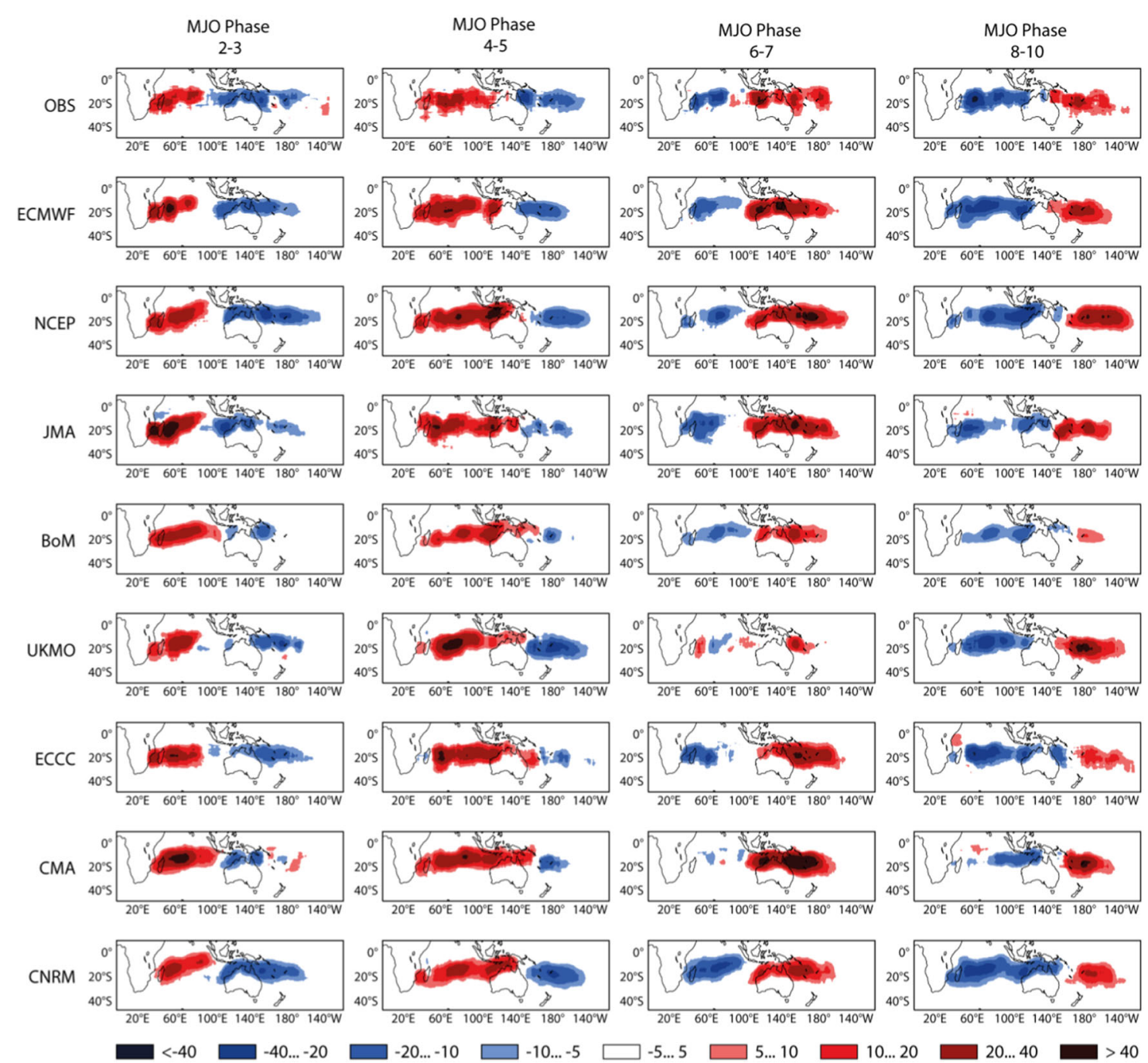

Fig. 2 Tropical cyclone density anomalies. Tropical storm density anomalies during the period October to March 1999-2010 when there is an MJO in phase 2 or 3 (left), phase 4 or 5 (middle left), phase 6 or 7 (middle right) and phase 8 or 1 (right). The top panels show observations (from Joint Typhoon Warning Center), the other panels show the tropical storm density anomalies from the reforecasts from ECMWF, NCEP, JMA, BoM, UKMO, CMA, ECCC and CNRM. The tropical storm density is calculated by computing the number of tropical storms passing within $500 \mathrm{~km}$ and by normalizing that number by the total number of tropical storms over the whole basin

predicted well the time evolution of this extreme event at the time range day $1-7$. At the time range day $8-14$, the model predicted well the timing of the intensification and the extreme values during the first 2 weeks of August, but it predicted the decay of the heat wave a week too late. In the extended range, at lead time day $15-21$, the model also predicted the day a week too late, and the intensity during the first 2 weeks of August was underestimated. However, the model at this time range was still able to predict an anomaly of 2-m temperature above the 95th percentile at the peak of the event, with some ensemble members above the 99th percentile. Figure 5, which focuses on the verifying week 1-7 August 2010, confirms that at the time range day 15-21 most ensemble members where in the 99th percentile, and even at a longer lead times (day 19-15) the ECMWF extended forecasts indicated some high risk of extreme 2-m temperature anomalies. Even if all ensemble members under-predicted the intensity taking place that week, the ECMWF extended range could have provided useful guidance at least 3 weeks in advance that 2-m temperature anomalies would be exceptionally high at large spatial scale over Russia.

The same study was performed with the other re-forecasts from the S2S database (Not Shown). At least 3 of other S2S models
(CNRM, JMA and BoM) also indicated a high probability of 2-m temperature within the 99th percentile about 3 weeks in advance. For some other S2S models, which have very small re-forecast ensemble size, this probability was difficult to estimate. Overall, these results suggest that sub-seasonal forecasts could be potentially useful for early warnings of such extreme events.

\section{ATTRIBUTION OF EXTREME EVENTS}

Another important area where S2S forecasts could be useful is in the attribution of the causes of extreme events to physical phenomena. S2S forecasting systems run in real-time quite frequently (at least once a week) with often large ensemble size and therefore produce a very extensive data set which can be used to better understand the origin of some extreme events. This attribution can be performed in several ways. Differences between ensemble members which successfully predicted an extreme event and those which did not may help better understand the causality of the extreme event.

For example, March 2013 was exceptionally cold over part of western Europe (second coldest month since 1900 over the United Kingdom), most of Russia and part of North America (Fig. 6, top 
READY

1 November 2010 seasonal forecast

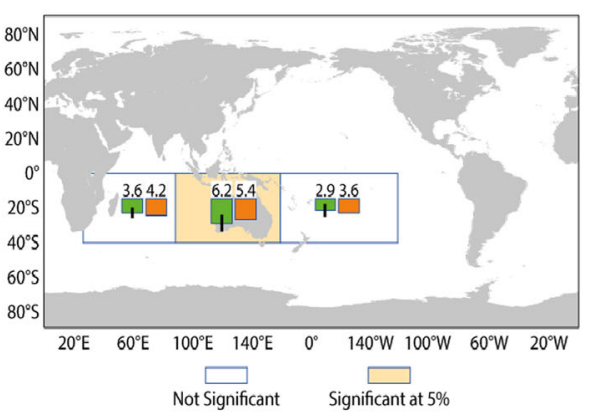

$\square$ Forecast mean - Standard deviation $\square$ Climate mean
SET

13 January 2011- Day 19-25

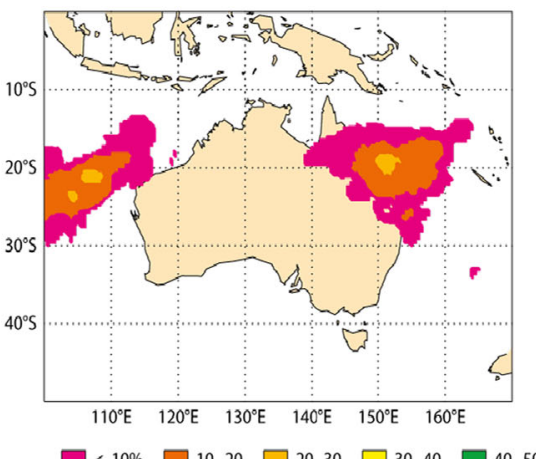

GO

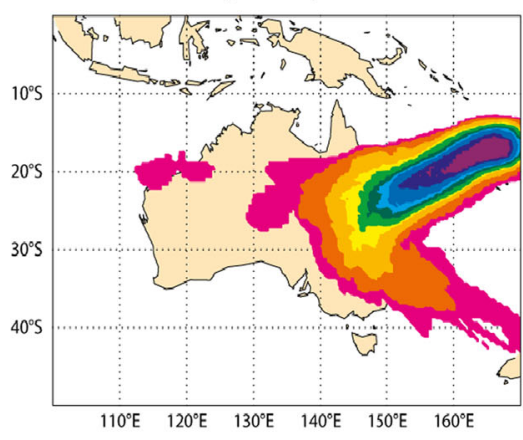

$60.60 .70 \square 70 . .80 \square 80.90 \square>90 \%$

Fig. 3 Tropical cyclone YASI (26 January-4 February 2011). Prediction of tropical cyclones at different time ranges from ECMWF seasonal and ensemble forecasting systems. The left panel shows a seasonal forecast of hurricane frequency starting on 1 st November 2010 and covering the period December 2010 to May 2011. Green bras represent the predicted frequency of hurricane-intensity cyclones compared to observed climatology (orange). The black bars show the $5 \%$ level of confidence. The middle panel shows the probability of a tropical cyclone strike within $300 \mathrm{~km}$ from the ECMWF extended range forecast starting on 13 January 2011 at the time range day 19-25, and the right panel the strike probabilities from the ECMWF medium range forecast starting on 27 January 2011 at the time range day 5-11

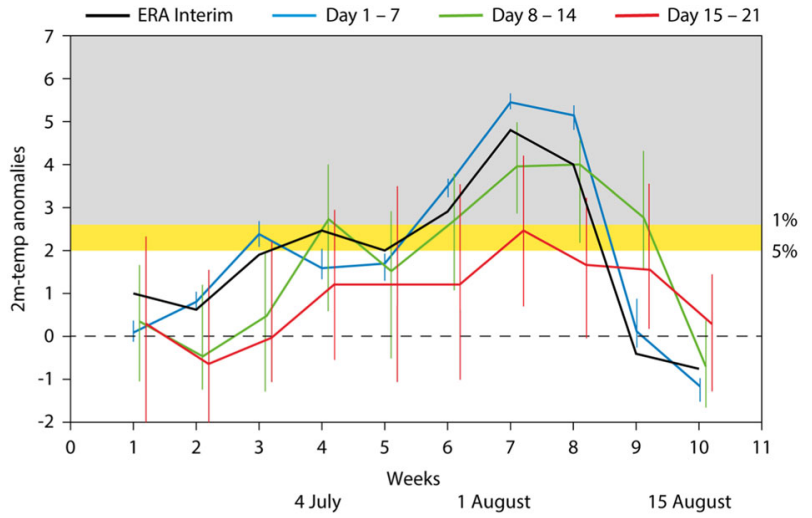

Fig. 42010 Russian heat wave prediction. Weekly mean 2-m temperature anomalies ensemble predictions from the ECMWF extended range forecasts for different lead times: day 1-7 (blue), day 8-14 (green), and day 15-21 (red curve) from mid-June to midAugust 2010. The vertical lines represent the ensemble spread. The black curve represents the verification from ERA Interim. The yellow and gray background represent respectively the 5 and 1 percentile from ERA Interim.

right panel). This event coincided with a strong MJO event propagating into the western Pacific (top left panel in Fig. 6). The 16-member extended range ensemble forecasts from NCEP (CFS. v2) starting from 14 February 2013 could be classified into two categories: those which predicted that the MJO event which was located in Phase 3 (Indian Ocean) on 14 February 2013 will propagate into the western Pacific, and the others (about half the ensemble members) which predicted that the MJO will die over the Maritime Continent. The 2-m temperature anomalies produced from the ensemble members with a fairly strong MJO produced a 2-m temperature anomaly consistent with ERA Interim, whereas the 2-m temperature anomalies produced from the ensemble members with no strong MJO in Phase 6 or 7 looks very different from the verification. This result suggests that this MJO event and the cold wave over part of the northern Hemisphere were linked, although the causality (is it the MJO which impacted the northern Hemisphere weather or the other way around?) would need further investigation.

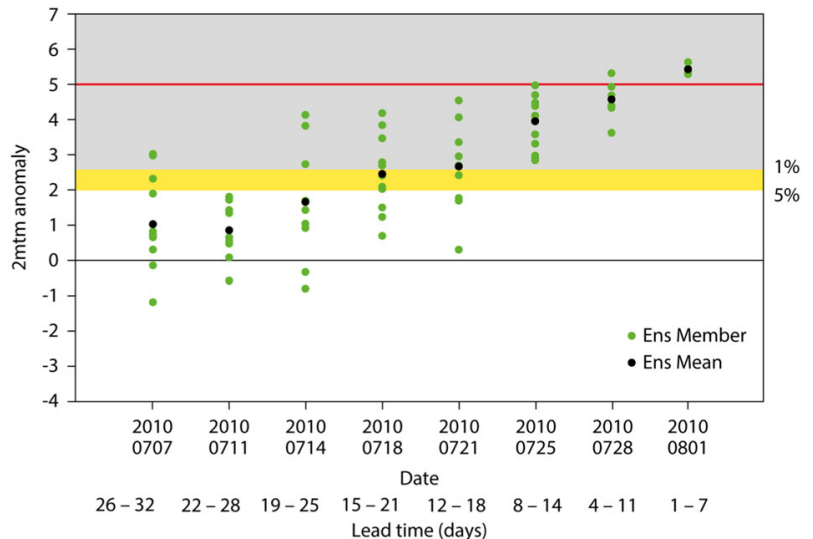

Fig. 5 2-m temperature anomalies forecast over Russia (1-7 August 2010). Weekly mean $2-m$ temperature anomalies ensemble predictions from the ECMWF extended range forecasts for different lead times and verifying on the week of 1-7 August 2010. The 2-m temperature has been average over the area $20 \mathrm{E}-50 \mathrm{E}, 45 \mathrm{~N}-70 \mathrm{~N}$. Each green point represents one of the 11 ensemble members. The black dots represent the ensemble means. The $x$-axis indicates the start dates and the forecast lead time in days. The anomalies have been computed relative to the model climatology. The yellow and gray background represent the 5 and 1, respectively, percentile from ERA Interim. The red line indicates the anomalies from ERA Interim

\section{CONCLUSIONS}

Sub-seasonal forecasting is still in its infancy, and certainly much less well understood and developed than medium range and seasonal forecasting. Sub-seasonal predictions have the potential to be useful to predict the onset, evolution and decay of some large scale, multi-week events such as long-lasting heat waves and droughts, as well as to predict changes in the probabilities of the occurrence of shorter lived extreme weather events, ranging from tornedoes to tropical cyclones, linked to changes in the large-scale circulation and environment. The examples presented above suggest that there is potential to use these forecasts for early warnings of several types of extreme events and also for better understanding the origin of some extreme events. Increased 

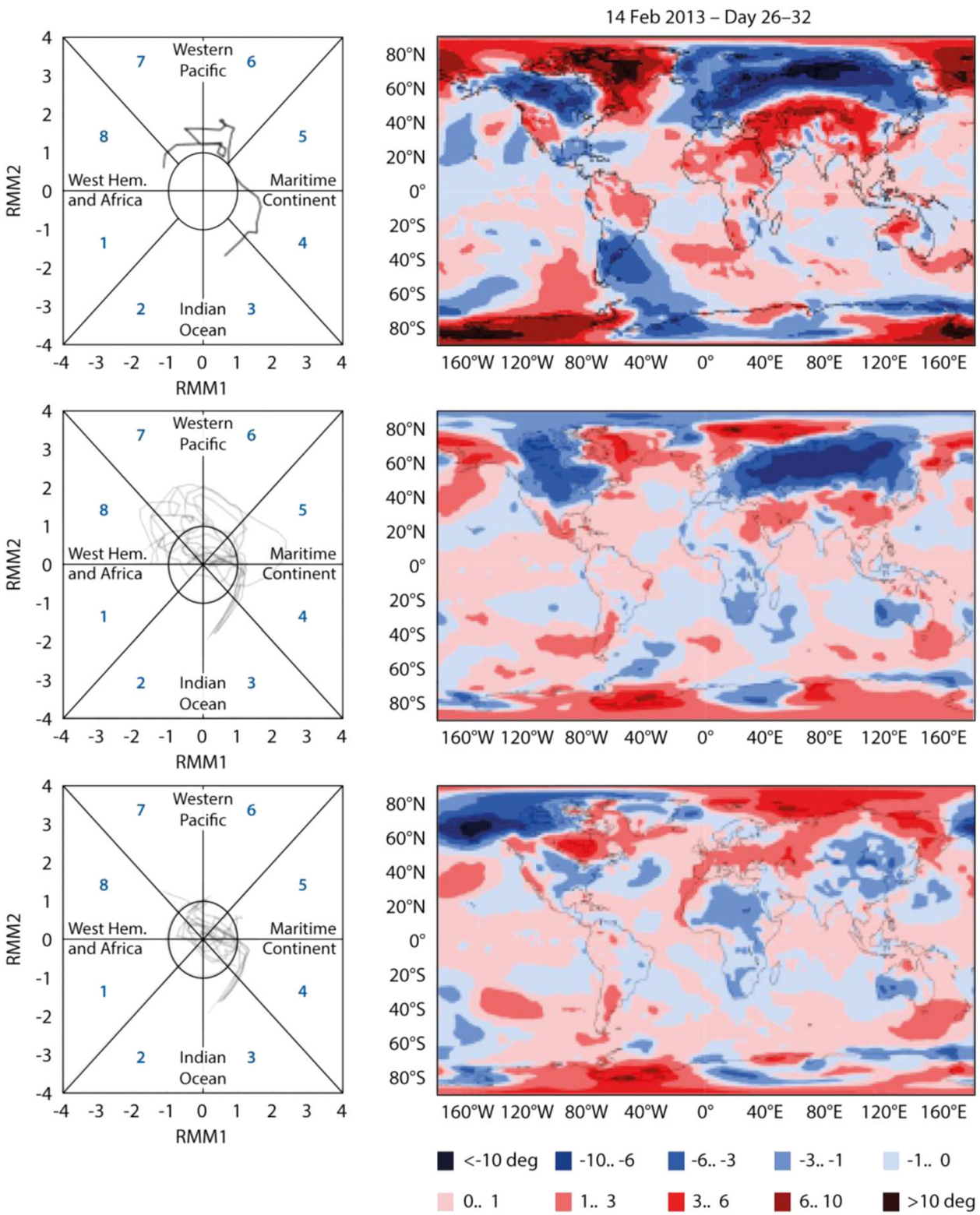

Fig. 6 Cold March 2013 Case Study. Weekly mean 2-m temperature anomalies (right panels) for the verifying week of 7-13 March 2013 from ERA Interim (top panel), ensemble mean of all the NCEP forecasts starting on 14 February 2013 (lead time day 26-32) which displayed a strong MJO (middle panel) and NCEP forecasts with a weak MJO (bottom panel). The cold anomalies over North America and Asia (middle right panel) are statistically significant within the $5 \%$ level of confidence. The left panel shows the MJO forecasts using the Wheeler and Hendon index (2004) from 14 February to 14 March 2013 from ERA interim (top), the 8 ensemble members from NCEP with the strongest MJOs (middle panel) and 8 weakest MJOs (bottom panel)

resolution, in addition to improved parametrizations, initialization schemes and continuous improvements in the prediction of major sources of sub-seasonal prediction ${ }^{23}$ should lead to a better representation of these extreme events, most especially the smaller scale ones like tropical cyclones, in operational subseasonal forecasting systems and more skillful S2S predictions.

Sub-seasonal forecasts of extreme events are starting to become available from some operational centers, such as weekly outlooks of tropical cyclone activity from ECMWF. Much work is still needed to fully integrate these forecasts into a fully integrated ready-set-go type of system, tailored for specific applications. An important goal of the S2S project is to create a few regional projects to demonstrate and quantify the benefits of using subseasonal forecasts of extreme events. This will be one of the foci of the next phase of the S2S project.
Data Availability

The data sets analyzed during the current study are available from the S2S database at s2s.ecmwf.int or s2s.cma.cn.

\section{ACKNOWLEDGEMENTS}

This work is based on S2S data. S2S is a joint initiative of the World Weather Research Programme (WWRP) and the World Climate Research Programme (WCRP). The original S2S database is hosted at ECMWF as an extension of the TIGGE database. A. W. Robertson acknowledges the support of NOAA's Modeling, Analysis, Predictions and Projections (MAPP) Program, grant NA16OAR4310145".

\section{AUTHOR CONTRIBUTIONS}

F.V. led the writing of the paper and produced Figs. 1-6. All authors contributed to writing the manuscript. 


\section{ADDITIONAL INFORMATION}

Competing interests: The authors declare no competing financial interests.

Publisher's note: Springer Nature remains neutral with regard to jurisdictional claims in published maps and institutional affiliations.

\section{REFERENCES}

1. NOAA Billion-Dollar Weather and Climate Disasters. National Oceanic and Atmospheric Administration, National Climatic Data Center (2013).

2. Munich Re, Press release, Available at: https://www.munichre.com/en/mediarelations/publications/press-releases/2011/2011-01-03-press-release/index.html (2011).

3. Coughlan de Perez, E. et al. Action-based flood forecasting for triggering humanitarian action, Hydrol. Earth Syst. Sci. 20, 3549-3560 (2016).

4. Vitart, F., Robertson A. and the S2S steering group Sub-seasonal to seasonal prediction: linking weather and climate. Seamless prediction of the earth system: from minutes to months, WMO-no 1156, 385-405 (2015).

5. Vitart, F., Robertson, A. \& Anderson, D. Sub-seasonal to Seasonal Prediction Project: bridging the gap between weather and climate. WMO Bull. 61, 23-28 (2012).

6. Vitart, F. et al. The Subseasonal to Seasonal (S2S) Prediction Project Database. Bull. Am. Meteor. Soc., https://doi.org/10.1175/BAMS-D-16-0017.1 (2017).

7. Bougeault, $P$. et al. The THORPEX interactive grand global ensemble (TIGGE). Bull. Am. Met. Soc. 91, 1059-1072 (2010).

8. Vitart, F. \& Stockdale, T. N. Seasonal forecasting of tropical storms using coupled GCM integrations. Mon. Weather Rev. 129, 2521-2527 (2001).

9. Camargo, S. J. \& Barnston, A. G. Experimental dynamical seasonal forecasts of tropical cyclone activity at IRI. Weather Forecast. 24, 472-491 (2009).

10. Knutson, T. R. et al. Global projections of intense tropical cyclone activity for the late twenty-first century from dynamical downscaling of CMIP5/RCP4.5 scenarios. J. Clim. 28, 7203-7224 (2015).

11. Kurihara, Y., Tuleya, R. E. \& Bender, M. A. The GFDL hurricane prediction system and its performance in the 1995 hurricane season. Mon. Weather Rev. 126, 1306-1322 (1998).

12. Yamaguchi, M. et al. Global distribution on the skill of tropical cyclone activity forecasts from short- to medium-range time scales. Weather Forecast. 30, 1695-1709 (2015).

13. Gray, W. M. in Supplement to Meteorology Over the Tropical Oceans, (ed. Shaw D. B.) 155-218 (James Glaisher House, Grenville Place, Bracknell, Berkshire, 1979).

14. Belanger, J. I., Webster, P. J., Curry, J. A. \& Jelinek, M. T. Extended prediction of north indian ocean tropical cyclones. Weather Forecast. 27, 757-769 (2012).

15. Leroy, A. \& Wheeler, M. C. Statistical prediction of weekly tropical cyclone activity in the southern hemisphere. Mon. Weather Rev. 136, 3637-3654 (2008).

16. Nakazawa, T. J. Intraseasonal variations of OLR in the tropics during the FGGE year. Meteor. Soc. Jpn. 64, 17-34 (1986).
17. Camargo, S. J., Wheeler, M. C. \& Sobel, A. H. Diagnosis of the MJO modulation of tropical cyclogenesis using an empirical index. J. Atmos. Sci. 66, 3061-3074 (2009).

18. Maloney, E. D. \& Hartmann, D. L. Modulation of eastern North Pacific hurricanes by the Madden-Julian oscillation. J. Clim. 13, 1451-1460 (2000).

19. Frank, W. M. \& Roundy, P. E. The relationship between tropical waves and tropical cyclogenesis. Mon. Weather Rev. 134, 2397-2417 (2006).

20. Gottschalk, J. et al. Framework for assessing operational model MJO forecasts: a project of the CLIVAR Madden-Julian Oscillation Working Group. Bull. Am. Meteor. Soc. 91, 1247-1258 (2010).

21. Wheeler. \& Hendon, H. H. An all-season real-time multivariate MJO index: Development of an index for monitoring and prediction. Mon. Weather Rev. 132, 1917-1932 (2004).

22. Rashid, H. A., Hendon, H. H., Wheeler, M. C. \& Alves O. Predictability of the Madden-Julian Oscillation in the POAMA Dynamical seasonal prediction system. Climate Dyn., https://doi.org/10.1007/s00382-010-0754-x (2010).

23. Vitart, F. Evolution of ECMWF sub-seasonal forecast skill scores. Quart. J. Roy. Meteor. Soc. 140, 1889-1899 (2014).

24. Vitart, F., Anderson, J. L. \& Stern, W. F. Simulation of interannual variability of tropical storm frequency in an ensemble of GCM integrations. J. Clim. 10, 745-760 (1997).

25. Vitart, F. Impact of the Madden Julian Oscillation on tropical storms and risk of landfall in the ECMWF forecast system. Geophys. Res. Lett. 36, L15802 (2009).

26. Vitart, F., Leroy, A. \& Wheeler, M. C. A comparison of dynamical and statistical predictions of weekly tropical cyclone activity in the Southern Hemisphere. Mon. Weather Rev. 138, 3671-3682 (2010).

27. Belanger, J. I., Curry, J. A. \& Webster, P. Predictability of North Atlantic Tropical Cyclone Activity on Intraseasonal Time Scales. Mon. Weather Rev. 138, 4362-4374 (2010).

28. Hoag, H. Russian summer tops 'universal' heatwave index. Nature News, https:// doi.org/10.1038/nature.2014.16250 (2014).

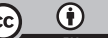

Open Access This article is licensed under a Creative Commons Attribution 4.0 International License, which permits use, sharing, adaptation, distribution and reproduction in any medium or format, as long as you give appropriate credit to the original author(s) and the source, provide a link to the Creative Commons license, and indicate if changes were made. The images or other third party material in this article are included in the article's Creative Commons license, unless indicated otherwise in a credit line to the material. If material is not included in the article's Creative Commons license and your intended use is not permitted by statutory regulation or exceeds the permitted use, you will need to obtain permission directly from the copyright holder. To view a copy of this license, visit http://creativecommons. org/licenses/by/4.0/.

(c) The Author(s) 2018 